\title{
Generalized Uncertainty Principles associated with One-Dimensional Hilbert Transform on LCT
}

\author{
ZHOU Jianjun ${ }^{1, a}$, CHEN Jie ${ }^{1, a}$ and XU Guanlei ${ }^{3, b^{*}}$ \\ ${ }^{1}$ Naval Academy of Armament, Beijing of China, 102200 \\ ${ }^{2}$ Department of Oceanography of Dalian Naval Academy, Dalian of China, 116018 \\ a13810605878@139.com, bxgl_86@163.com
}

\begin{abstract}
Keywords: Uncertainty Principle; Linear Canonical Transform(LCT); Generalized Hilbert Transform (GHT); Complex Signals
\end{abstract}

\begin{abstract}
This paper discusses the Heisenberg's uncertainty principle and windowed uncertainty principle associated with 1D linear canonical transform (LCT) and the Heisenberg's uncertainty principle associated with two types of 2D LCT for the first time. These uncertainty bounds are related with the transform parameters of LCT. Also, we show that the certainty bounds for the complex signals derived by LCT are different with that for the general complex signals in most cases. The special cases of these principles in traditional domains are provided as well. On one hand, these new uncertainty relations enrich the ensemble of uncertainty principles, and on the other hand, these derived bounds yield new understanding of complex signals via Hilbert transform.
\end{abstract}

\section{Introduction}

Heisenberg's uncertainty principle plays an important role in mathematics, physics, signal processing and information theory and so on. There have been many extensions [1-13] of traditional Heisenberg's uncertainty principle [14-16] in these fields or new transform domains. Recently, there are many papers, such as [2-13], involving new various uncertainty principles in new transform domains. However, none of them has covered the uncertainty principles associated with Hilbert transform in linear canonical transform (LCT) domain, that is to say, the uncertainty principle for the complex signals derived by Hilbert transform have not been involved despite of some discussions of Heisenberg's uncertainty principle on new transform for general complex signals $[1,3,4,9,12,13]$.

Hilbert transform $[14,15]$ is an elementary tool in signal analysis and is of very much importance to communication and time-frequency analysis. The complex signal, derived from Hilbert transform, is different from the general complex signal and widely used in many fields such as communication, optics and radar theory. Therefore, there is great need to discuss the uncertainty principles for the complex signals derived by Hilbert transform. In this paper, we will discuss the uncertainty principles for the complex signals derived by Hilbert transform in 1D and 2D LCT domains. Without loss of generality and for the sake of simpliness, we assume that all the means are zeros in any domains in this paper.

As the generalization of the traditional Fourier transform (FT), FRFT [16, LCT [16] has some properties with its transform parameter and sometimes gives better signal analysis results. More details can be found in [16]. Here we only briefly review its definition.

1D LCT is the generalization of the classical 1D Fourier transform. The LCT of one real function $f(x, y)(x, y \in \mathfrak{R})$ is defined as follow:

$$
F^{A}\{f(x)\}=F^{A}(u)=\int_{-\infty}^{+\infty} f(x) K_{A}(x, u) d x=\left\{\begin{array}{ll}
\frac{1}{2 \pi i} \sqrt{\frac{1}{b}} \cdot e^{i\left(\frac{d u^{2}}{2 b}\right)} \int_{-\infty}^{+\infty} e^{-i \frac{u x}{b}} e^{i \frac{a x^{2}}{2 b}} f(x) d x \quad b \neq 0,|A|=1 \\
\sqrt{d} e^{i \frac{c d u^{2}}{2}} f(d u) & b=0
\end{array},\right.
$$


where, $K_{A}(x, u)=\sqrt{\frac{1}{2 \pi i b}} \cdot e^{i \frac{d u^{2}}{2 b}} e^{-i \frac{u x}{b}} e^{i \frac{a x^{2}}{2 b}}, A=\left[\begin{array}{ll}a & b \\ c & d\end{array}\right]$, and $A$ are real matrices. $A^{-1}=\left[\begin{array}{cc}-d & -b \\ -c & a\end{array}\right]$. In this paper we assume that the real function $f(x)(x \in \mathfrak{R})$ is absolute integrable both in $\mathrm{X}$ and $\mathrm{Y}$ directions and satisfies the Dirichlet condition. In addition it satisfies the Fubini's theorem. In particular, if $A=\left[\begin{array}{cc}0 & 1 \\ -1 & 0\end{array}\right],(1)$ is 1D Fourier transform multiplied by $-i$.If $A=\left[\begin{array}{cc}0 & -1 \\ 1 & 0\end{array}\right],(1)$ is 1D reversed Fourier transform multiplied by $i[16]$.

The paper is organized as follows. Section 2 yields the Heisenberg's uncertainty principle and the windowed uncertainty principle associated one 1D LCT. In section 3 the Heisenberg's uncertainty principles for general complex signals and the Heisenberg's uncertainty principles for the complex signals associated with two 2D LCT are discussed. The last section is the conclusion of this paper.

\section{The Principle of RSA Algorithm}

\subsection{D Generalized Hilbert Transform}

There are some new GHT [17-21] up till now. However, in this paper we will use the definition in [26] because it has similar physical sense with that of the traditional counterpart, and in the future we will extend the uncertainty principle to other GHT [18-21]. For a real function $f(t)(t \in R)$, the 1D GHT is defined as follows:

$$
f^{H_{A}}(t)=H_{A}\{f(t)\}=\frac{e^{-i \frac{a t^{2}}{2 b}}}{\pi} \int_{-\infty}^{+\infty} \frac{f(\xi) e^{i \frac{\xi^{2}}{2 b}}}{x-\xi} d \xi \text {, where } A=\left[\begin{array}{ll}
a & b \\
c & d
\end{array}\right] .
$$

The according analytic signal is defined as:

$$
f_{H_{A}}(t)=f(t)+i f^{H_{A}}(t) .
$$

The according LCT of $f_{H_{A}}(t)$ is

$$
F_{H_{A}}(u)=F_{A}(u)+i\left(-i \operatorname{sgn} u F_{A}(u)\right)=2 F_{A}(u) \quad \text { for } u>0 .
$$

Therefore, this physical sense here is clearly the same as that of the traditional counterpart: the GHT in LCT domain obtains its analytic signal by suppressing the negative components and doubling the positive components. In addition, if $A=\left[\begin{array}{cc}0 & -1 \\ 1 & 0\end{array}\right]$, the definition reduces to the traditional Hilbert transform. In the next we will use this definition in (2)-(4) to explore the uncertainty principles.

\subsection{Uncertainty Principles on 1D Linear Canonical Transform}

In this section we will discuss the uncertainty principles on 1D LCT. Before beginning, we first introduce a lemma defined in [16].

Lemma 1: If $f(t) \in L^{1}(R) \cap L^{2}(R)$ and $f(t)$ is odd, $F(u)$ is the Fourier transform of $f(t)$, then

$$
\left(\int_{-\infty}^{\infty}|t f(t)|^{2} d t / \int_{-\infty}^{\infty}|f(t)|^{2} d t\right) \cdot\left(\int_{-\infty}^{\infty}|u F(u)|^{2} d u / \int_{-\infty}^{\infty}|F(u)|^{2} d u\right) \geq 9 / 4
$$

The detailed content can be found in [16]. Based on this lemma, we give another lemma by us in the follows.

Lemma 2: If $f(t) \in L^{1}(R) \cap L^{2}(R)$ and $f(t)$ is odd, $F_{A}(u), F_{B}(v)$ are the LCTs of $f(t)$ with transform parameters $A=\left[\begin{array}{ll}a_{1} & b_{1} \\ c_{1} & d_{1}\end{array}\right]$ and $B=\left[\begin{array}{ll}a_{2} & b_{2} \\ c_{2} & d_{2}\end{array}\right]$, then

$$
\left(\int_{-\infty}^{\infty}\left|u F_{A}(u)\right|^{2} d u / \int_{-\infty}^{\infty}\left|F_{A}(u)\right|^{2} d u\right) \cdot\left(\int_{-\infty}^{\infty}\left|v F_{B}(v)\right|^{2} d v / \int_{-\infty}^{\infty}\left|F_{B}(v)\right|^{2} d v\right) \geq 9\left(a_{1} b_{2}-a_{2} b_{1}\right)^{2} / 4 \text {. }
$$

Proof: First, without loss of generalization we assume $b_{1}^{2}+b_{2}^{2}+b^{2}=0$ and $C=\left[\begin{array}{ll}a & b \\ c & d\end{array}\right]$. 
Set $G(u)=F_{A}(u) \exp \left(-i \frac{a_{1} u^{2}}{2 b_{1}}\right), F_{A}(u)=F_{A}(f(t)), g(t)=\sqrt{\frac{1}{2 \pi}} \int_{-\infty}^{\infty} G(u) e^{i u t} d u$, and note the fact that $\left|F_{A}(u) \exp \left(-i \frac{a_{1} u^{2}}{2 b_{1}}\right)\right|=\left|F_{A}(u)\right|$ (because $\left.\left|\exp \left(-i \frac{a_{1} u^{2}}{2 b_{1}}\right)\right|=1\right)$ holds, we get

$$
\begin{aligned}
& \left(\int_{-\infty}^{\infty}|\operatorname{tg}(t)|^{2} d t / \int_{-\infty}^{\infty}|g(t)|^{2} d t\right) \cdot\left(\int_{-\infty}^{\infty}|u G(u)|^{2} d u / \int_{-\infty}^{\infty}|G(u)|^{2} d u\right) \\
& =\left(\int_{-\infty}^{\infty}|\operatorname{tg}(t)|^{2} d t / \int_{-\infty}^{\infty}|g(t)|^{2} d t\right) \cdot \int_{-\infty}^{\infty}\left|u F_{A}(u)\right|^{2} d u / \int_{-\infty}^{\infty}\left|F_{A}(u)\right|^{2} d u \geq 9 / 4 .
\end{aligned}
$$

Noting $g(t)$, we get $\left(\int_{-\infty}^{\infty}|\operatorname{tg}(t)|^{2} d t / \int_{-\infty}^{\infty}|g(t)|^{2} d t\right)=\frac{1}{|b|} \int_{-\infty}^{\infty}\left|\frac{t}{b}\right|^{2}\left|g\left(\frac{t}{b}\right)\right|^{2} d t$.

Here from the definition of FT we have $\left|g\left(\frac{t}{b}\right)\right|^{2}=\left|\sqrt{\frac{1}{2 \pi}} \int_{-\infty}^{\infty} G(u) e^{i \frac{u t}{b}} d u\right|^{2}$.

Substituting $F_{A}(u) \exp \left(-i \frac{a u^{2}}{2 b}\right)$ for $G(u)$ and using the definition of LCT, we get

$$
\left|g\left(\frac{t}{b}\right)\right|^{2}=|b| \cdot\left|F_{C^{-1}}\left\{F_{A}(u)\right\}(t)\right|^{2} \text {. }
$$

Thus we obtain

$$
\int_{-\infty}^{\infty}|\operatorname{tg}(t)|^{2} d t / \int_{-\infty}^{\infty}|g(t)|^{2} d t=\frac{1}{|b|} \int_{-\infty}^{\infty}\left|\frac{t}{b}\right|^{2}\left|g\left(\frac{t}{b}\right)\right|^{2} d t=\frac{1}{\mid b} \int_{-\infty}^{\infty}\left|\frac{t}{b}\right|^{2}\left(|b| \cdot\left|F_{C^{-1}}\left\{F_{A}(u)\right\}(t)\right|^{2}\right) d t .
$$

Set $t=v$ and from the relation $F_{C^{-1}}\left\{F_{A}(u)\right\}(t)=F_{A C^{-1}}(u)$, have

$$
\int_{-\infty}^{\infty}|\operatorname{tg}(t)|^{2} d t / \int_{-\infty}^{\infty}|g(t)|^{2} d t=\frac{1}{|b|^{2}} \int_{-\infty}^{\infty}\left|v F_{A C^{-1}}(v)\right|^{2} d v .
$$

Set $B=A C^{-1}$, then we have $\frac{\int_{-\infty}^{\infty}|\operatorname{tg}(t)|^{2} d t}{\int_{-\infty}^{\infty}|g(t)|^{2} d t}=\frac{1}{\left|a_{1} b_{2}-a_{2} b_{1}\right|^{2}} \int_{-\infty}^{\infty}\left|v F_{B}(v)\right|^{2} d v$.

We obtain

$$
\left(\int_{-\infty}^{\infty}\left|u F_{A}(u)\right|^{2} d u / \int_{-\infty}^{\infty}\left|F_{A}(u)\right|^{2} d u\right) \cdot\left(\int_{-\infty}^{\infty}\left|v F_{B}(v)\right|^{2} d v / \int_{-\infty}^{\infty}\left|F_{B}(v)\right|^{2} d v\right) \geq 9\left(a_{1} b_{2}-a_{2} b_{1}\right)^{2} / 4 .
$$

The same result can also be obtained via the relation between FT and LCT [22].

$$
\text { If } A=\left[\begin{array}{ll}
1 & 0 \\
0 & 1
\end{array}\right], \text { Eq. (6) reduces to }\left(\int_{-\infty}^{\infty}|t f(t)|^{2} d t / \int_{-\infty}^{\infty}|f(t)|^{2} d t\right) \cdot\left(\int_{-\infty}^{\infty}\left|v F_{B}(v)\right|^{2} d v / \int_{-\infty}^{\infty}\left|F_{B}(v)\right|^{2} d v\right)
$$

$\geq 9 b_{2}^{2} / 4$. If $A=\left[\begin{array}{ll}1 & 0 \\ 0 & 1\end{array}\right], B=\left[\begin{array}{rr}0 & 1 \\ -1 & 0\end{array}\right],(6)$ reduces to the traditional case. In addition, if $\left|a_{1} b_{2}-a_{2} b_{1}\right|=1$, the bound of (6) reduces to 9/4, which shows that the two LCTs are orthogonal.

Theorem 1: If $f(t) \in L^{1}(R) \cap L^{2}(R)$ and $f(t)$ is odd, $f_{H_{A}}(t)$ and $f_{H_{B}}(t)$ are the generalized complex signals obtained by the LCT of $f(t)$ defined in with transform parameters $A=\left[\begin{array}{ll}a_{1} & b_{1} \\ c_{1} & d_{1}\end{array}\right]$, $B=\left[\begin{array}{ll}a_{2} & b_{2} \\ c_{2} & d_{2}\end{array}\right], F_{H_{A}}(u)$ and $F_{H_{B}}(v)$ are the LCTs of $f_{H_{A}}(t)$ and $f_{H_{B}}(t)$ with transform parameters $A, B$, respectively, then

$$
\left(\int_{-\infty}^{\infty}\left|u F_{H_{A}}(u)\right|^{2} d u / \int_{-\infty}^{\infty}\left|F_{H_{A}}(u)\right|^{2} d u\right) \cdot\left(\int_{-\infty}^{\infty}\left|v F_{H_{B}}(v)\right|^{2} d v / \int_{-\infty}^{\infty}\left|F_{H_{B}}(v)\right|^{2} d v\right) \geq 9\left(a_{1} b_{2}-a_{2} b_{1}\right)^{2} / 4
$$

Proof: From the definition of LCT, we know that 


$$
F_{H_{A}}(u)=\left\{\begin{array}{ll}
2 F_{A}(u) & u>0 \\
F_{A}(u) & u=0 . \\
0 & u<0
\end{array} .\right.
$$

Since $f(t)$ is odd and $\int_{0-}^{0+}|s(x)|^{2} d x=0$ for $s(0)<\infty$, then $F_{A}(u)$ is odd and we obtain

$$
\begin{aligned}
& \int_{-\infty}^{\infty}\left|F_{H_{A}}(u)\right|^{2} d u=\int_{-\infty}^{0-}|0|^{2} d u+\int_{0-}^{0+}\left|F_{A}(u)\right|^{2} d u+\int_{0+}^{\infty}\left|2 F_{A}(u)\right|^{2} d u \\
& =2\left(-\int_{0-}^{-\infty}\left|F_{A}(-u)\right|^{2} d u+\int_{0-}^{0+}\left|F_{A}(u)\right|^{2} d u+\int_{0+}^{\infty}\left|F_{A}(u)\right|^{2} d u\right) \\
& =2\left(\int_{-\infty}^{0-}\left|F_{A}(u)\right|^{2} d u+\int_{0-}^{0+}\left|F_{A}(u)\right|^{2} d u+\int_{0+}^{\infty}\left|F_{A}(u)\right|^{2} d u\right) \\
& =2 \int_{-\infty}^{\infty}\left|F_{A}(u)\right|^{2} d u .
\end{aligned}
$$

Similarly, we obtain $\int_{-\infty}^{\infty}\left|u F_{H_{A}}(u)\right|^{2} d u=2 \int_{-\infty}^{\infty}\left|u F_{A}(u)\right|^{2} d u$.

Therefore, we obtain $\int_{-\infty}^{\infty}\left|u F_{H_{A}}(u)\right|^{2} d u / \int_{-\infty}^{\infty}\left|F_{H_{A}}(u)\right|^{2} d u=\int_{-\infty}^{\infty}\left|u F_{A}(u)\right|^{2} d u / \int_{-\infty}^{\infty}\left|F_{A}(u)\right|^{2} d u$.

In the same manner, we obtain $\int_{-\infty}^{\infty}\left|u F_{H_{B}}(u)\right|^{2} d u / \int_{-\infty}^{\infty}\left|F_{H_{B}}(u)\right|^{2} d u=\int_{-\infty}^{\infty}\left|u F_{B}(u)\right|^{2} d u / \int_{-\infty}^{\infty}\left|F_{B}(u)\right|^{2} d u$.

Therefore the theorem is verified.

If $A=\left[\begin{array}{ll}1 & 0 \\ 0 & 1\end{array}\right]$, the bound in (7) reduces to $9 b_{2}^{2} / 4$. If $A=\left[\begin{array}{ll}1 & 0 \\ 0 & 1\end{array}\right], B=\left[\begin{array}{rr}0 & 1 \\ -1 & 0\end{array}\right]$, (7) reduces to the traditional case. In addition, if $\left|a_{1} b_{2}-a_{2} b_{1}\right|=1$, the bound of (7) reduces to 9/4, which shows that the two LCTs are orthogonal.

Theorem 2: If $f(t) \in L^{1}(R) \cap L^{2}(R)$ and $f(t)$ is odd, $f_{H_{A}}(t)$ and $f_{H_{B}}(t)$ are the generalized complex signals obtained by the GHT of $f(t)$ defined in [17] with transform parameters $A=\left[\begin{array}{ll}a_{1} & b_{1} \\ c_{1} & d_{1}\end{array}\right]$, $B=\left[\begin{array}{ll}a_{2} & b_{2} \\ c_{2} & d_{2}\end{array}\right], F_{H_{A}}(u)$ and $F_{H_{B}}(v)$ are the LCTs of $f_{H_{A}}(t)$ and $f_{H_{B}}(t)$ with transform parameters $A, B$, $\left(\Delta v_{H_{A}}^{2}\right)_{S T}$ and $\left(\Delta u_{H_{B}}^{2}\right)_{S T}$ are the definition of windowed generalized spreads in [3] for $f_{H_{A}}(t)$ and $f_{H_{B}}(t)$, then

$$
\left(\Delta v_{H_{A}}^{2}\right)_{S T} \cdot\left(\Delta u_{H_{B}}^{2}\right)_{S T} \geq 9\left(\left|a_{1} b_{2}-a_{2} b_{1}\right|+1\right)^{2} / 4 \text {. }
$$

The proof is similar with that in [3], which is trivial and ignored here.

If $A=\left[\begin{array}{ll}1 & 0 \\ 0 & 1\end{array}\right]$, the bound of (8) reduces to $9\left(\left|b_{2}\right|+1\right)^{2} / 4$. If $A=\left[\begin{array}{ll}1 & 0 \\ 0 & 1\end{array}\right], B=\left[\begin{array}{rr}0 & 1 \\ -1 & 0\end{array}\right]$, (8) reduces to the traditional case: $\left(\Delta v_{H}^{2}\right)_{S T} \cdot\left(\Delta u_{H}^{2}\right)_{S T} \geq 9$. In addition, if $\left|a_{1} b_{2}-a_{2} b_{1}\right|=1$, the bound of (8) reduces to 9 , which shows that the two LCTs are in the orthogonal domains.

\section{Conclusions}

We derive the uncertainty principles associated with GHT for the first time. In 1D case, we discuss the Heisenberg's uncertainty principle and the windowed uncertainty principle for the complex signals derived by GHT on LCT, which have different bounds with that of the general complex signals. On one hand, these new uncertainty relations enrich the ensemble of uncertainty principles, and on the other hand, these derived bounds yield new understanding of complex signals via Hilbert transform. In future, we will extend these results to discrete cases. 


\section{Acknowledgment}

This work is supported by NSFC projects (No. 61002052, 61471412), all support is gratefully acknowledged.

\section{References}

[1] S. Shinde, M. G.Vikram. An uncertainty principle for real signals in the fractional Fourier transform domain, IEEE Trans. Signal Process, vol.49, no.11, pp. 2545-2548, 2001.

[2] D. Mustard. Uncertainty principle invariant under fractional Fourier transform, J. Austral. Math. Soc. Ser. B, vol.33, pp.180-191, 1991.

[3] G.L. Xu, X.T. Wang, X.G. Xu. The logarithmic, Heisenberg's and short-time uncertainty principles associated with fractional Fourier transform, Signal Processing, vol.89, no.3,pp. 339-343,2009.

[4] Xu Guanlei, Wang Xiaotong, Xu Xiaogang. Generalized Uncertainty Principles associated with Hilbert Transform. Signal, Image and Video processing(preprinted).

[5] G.L. Xu, X.T. Wang, X.G. Xu. The Entropic Uncertainty Principle in Fractional Fourier Transform Domains, Signal Processing, 2009, 89 (12): 2692-2697.

[6] A. Stern. Sampling of linear canonical transformed signals, Signal Processing 86, no.7, pp.1421-1425,2006.

[7] G.L. Xu, X.T. Wang, X.G. Xu. Uncertainty inequalities for Linear Canonical Transform”, IET Signal Processing, vol.3, no.5, pp. 392-402, 2009.

[8] K.K. Sharma, S.D. Joshi. Uncertainty Principle for Real Signals in the Linear Canonical Transform Domains”, IEEE Trans. Signal Process,vol.56, no.7, pp.2677 - 2683,2008.

[9] G.L. Xu, X.T. Wang, X.G. Xu. Three Cases of Uncertainty Principle for Real Signals in Linear Canonical Transform Domain”, IET Signal Processing, vol.3, no.1,pp.85-92,2009.

[10] A. Stern. Uncertainty principles in Linear Canonical Transform domains and some of their implications in optics", J. Opt. Soc. Am. A, vol.25, no.3, pp. 647-652, 2008.

[11] Xu Guanlei, Wang Xiaotong, Xu Xiaogang. New Inequalities and Uncertainty Relations on Linear Canonical Transform Revisit , EURASIP Journal on Advances in Signal Processing, 2009. doi:10.1155/2009/563265

[12] Xu G., Wang X., Xu X.. On Uncertainty Principle for the Linear Canonical Transform of Complex Signals. IEEE Transactions on Signal Processing, 2010, 58(9): 4916-4918

[13] J. Zhao, R. Tao, Y. L. Li, and Y. Wang.Uncertainty Principles for Linear Canonical Transform”, IEEE Trans. Signal Process, vol.57, no. 7, pp.2856-2858, 2009.

[14] G.L. Xu, X.T. Wang, X.G. Xu. Generalized Hilbert transform and its properties in 2D LCT domain, Signal Processing, 2009, 89 (7):1395-1402.

[15] A. W. Lohmann, D. Mendlovic, Z. Zalevsky. Fractional Hilbert transform, Opt. Lett., 21(1996): $281-283$.

[16] R. Tao, B. Deng, Y. Wang. Theory and application of the fractional Fourier transform, Tsinghua University Press, Beijing, 2009. 